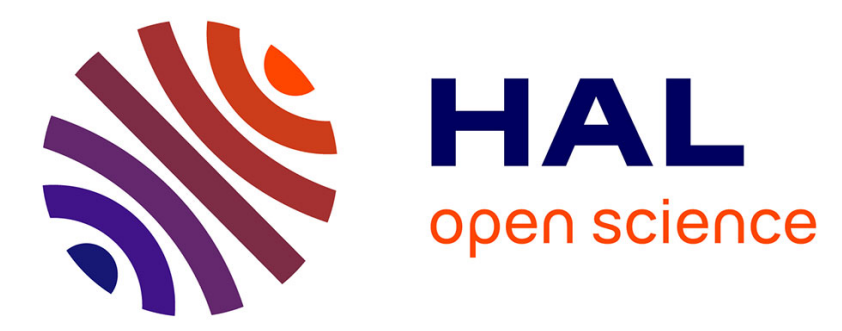

\title{
Jean-Claude Farcy, Dominique Kalifa et Jean-Noël Luc (dir.) L'enquête judiciaire en Europe au XIXe siècle. Acteurs, imaginaires, pratiques Paris, Créaphis, 2007, $386 \mathrm{p}$. \\ Martine Kaluszynski
}

\section{To cite this version:}

Martine Kaluszynski. Jean-Claude Farcy, Dominique Kalifa et Jean-Noël Luc (dir.) L'enquête judiciaire en Europe au XIXe siècle. Acteurs, imaginaires, pratiques Paris, Créaphis, 2007, 386 p. : COMPTE-RENDU ouvrage pour les ANNALES. 2012, pp 277-278. hal-00669333

\section{HAL Id: hal-00669333 https://hal.science/hal-00669333}

Submitted on 13 Feb 2012

HAL is a multi-disciplinary open access archive for the deposit and dissemination of scientific research documents, whether they are published or not. The documents may come from teaching and research institutions in France or abroad, or from public or private research centers.
L'archive ouverte pluridisciplinaire $\mathbf{H A L}$, est destinée au dépôt et à la diffusion de documents scientifiques de niveau recherche, publiés ou non, émanant des établissements d'enseignement et de recherche français ou étrangers, des laboratoires publics ou privés. 
Jean-Claude Farcy, Dominique Kalifa et Jean-Noël Luc (dir.)

L'enquête judiciaire en Europe au XIX $X^{e}$ siècle. Acteurs, imaginaires, pratiques

Paris, Créaphis, 2007, 386 p.

C'est à l'initiative de trois grands historiens, spécialistes de la justice, de la police et de la question pénale= que nous devons cet ouvrage issu d'un colloque organisé en 2005 par le Centre d'histoire du $\mathrm{XIX}^{\mathrm{e}}$ siècle et le Centre Georges Chevrier. Divisé en cinq parties, il rassemble plus d'une trentaine de contributions et démontre de façon magistrale comment l'enquête est à la fois un objet, une pratique, une technique, une posture et un mode d'information révélateurs de changements et de mutations dans une société qui accorde un rôle de plus en plus important aux savoirs et aux techniques. Disons-le d'emblée, l'aspect cumulatif, loin d'être un défaut, enrichit sans cesse le regard sur la complexité d'une pratique apparemment simple et moderne. L'ensemble donne le sentiment d'un puzzle qui se construit et offre au regard les multiples facettes de cet objet qui bascule entre son usage et sa représentation.

Dominique Kalifa montre dans son introduction que l'histoire contemporaine du crime s'est développée dans la périphérie même du phénomène. Il écrit « Le souci s'est cependant manifesté de dépasser ces approches fragmentées pour tenter de penser ensemble les multiples composantes du fait criminel et de restituer ainsi, dans toute son épaisseur sociale, un peu de la compréhension qui entourait le crime à une époque donnée »(p.5) et plus loin «C'est par le travail de l'enquête judiciaire et son processus d'élucidation progressive, qui devient peu à peu maîtrisable, qu'il advient comme « réalité » sociale »(p.5-6) . L’opération judiciaire rassemble police, justice, gendarmerie et «constitue $\underline{\mathrm{XIX}^{\mathrm{e}} \text { siècle un enjeu majeur de l'ordre social, un instrument de sa protection, un outil de sa }}$ reproduction $\gg($ p.9).

À la fin du XIX ${ }^{\mathrm{e}}$ siècle, la croissance industrielle et l'urbanisation ont bouleversé les modes d'existence, déstabilisant une frange importante de la population. La violence est perçue comme négative, le crime est le terrain privilégié pour refléter les peurs d'une société en mouvement, et l'homme moderne veut tout contrôler. On approche le fonctionnement des enquêtes et des pratiques judiciaires $\overline{\bar{y}}$ au cours d'un siècle souvent associé aux «progrès » de la rationalité judiciaire même si 
comme le dit D. Kalifa, «l'histoire des enquêtes judiciaires ne peut donc être lue comme celle d'une marche triomphante vers la rationalité légale et la certitude scientifique »(p.9)

Cet ouvrage est un voyage à travers des espaces variés : la France où sont sondées les évolutions institutionnelles autour de l'enquête pénale et civile, l'Angleterre dont Clive Emsley montre l'évolution feutrée mais déterminée, la Belgique inspirée par le modèle napoléonien, le Portugal que Luís Eloy Azevedo nous dépeint en période de transition et où ne surgissent pas encore les «grands enquêteurs », l'Italie où Marcella Marmo aborde les procès de la camorra au tribunal de Naples. C'est également une traversée de périodes politiques très diversifiées, qui constituent des configurations politiques et sociales particulières présentant des caractères spécifiques du point de vue de la mise en œuvre de l'enquête judiciaire : 1806 et l'instauration du code de procédure civile analysée par JeanJacques Clère, la monarchie de Juillet, ou les années 1880-1914, 1900-1930, etc...

L'enquête est abordée, construite, pratiquée par des acteurs multiples, tout aussi différents, restituant ainsi la totalité des étapes de la chaine pénale. On y voit les accusés, les fauteurs de troubles, les apaches, les incendiaires ou les jeunes délinquants, pour qui l'enquête sera remodelée afin de s'ajuster à ce profil spécifique, comme le montre Pascale Quincy-Lefebvre.

Mais le projecteur éclaire surtout les professionnels que sont les policiers et les gendarmes, que Caroline Broussaud montre en difficulté au cours de leurs «tournées de communes » requises par la «clameur publique» dont parle aussi Jean-Claude Caron. L'enquête judiciaire confronte ces gendarmes aux autres professionnels comme les magistrats ou juges d'instruction. On observe également le juge de paix, les gardes champêtres mais aussi les médecins dont on sait le rôle grandissant qu'ils ont acquis à la fin du $\mathrm{XIX}^{\mathrm{e}}$ siècle avec l'introduction de l'expertise dans les tribunaux, observée par Marina Daniel et Laurence Guignard. L'enquête judiciaire révèle ces professionnels dont le métier se transforme. On perçoit clairement les difficultés, les résistances (le cas des fonctionnaires de l'Assistance publique décrit par Yvan Jablonka) mais aussi l'acculturation et le changement d'éthos.

Ce sont les formes mêmes de l'enquête (ainsi les gendarmes confrontés au portrait parlé, dépeints par Arnaud-Dominique Houtte ou Aurélien Lignereux), la manière de recueillir les récits, les preuves avancées, qui sont remises en cause, portées par les techniques et les instruments qui se 
développent à cette époque en accord avec un modèle épistémologique que Carlo Ginzburg avait

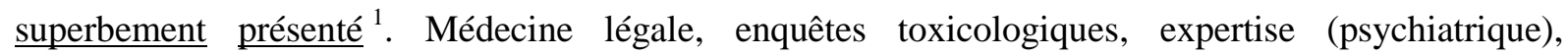
photographie «scientifique» deviennent les auxiliaires incontournables de la justice. Ils épaulent une

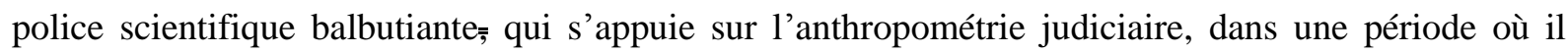
n'est plus question de marquer les coupables= et où l'identification devient la condition élémentaire de la répression, même si Ilsen About met parfaitement en lumière les difficultés d'appropriation et les rivalités présentes.

Cet ouvrage dévoile l'infinie étendue des possibilités d'une pratique ancrée dans un premier temps dans le domaine judiciaire, mais qui rapidement touche l'ensemble de la société. Ainsi, on aperçoit d'autres corps de métier comme les journalistes en recherche de vérité, étudiés par Olivier Isaac, dont on sait qu'ils ont développé depuis une compétence véritable, même concurrentielle avec les policiers dans de grandes affaires médiatiques et retentissantes en cette fin du XIX ${ }^{\mathrm{e}}$ siècle. Certaines sont évoquées : l'affaire Vacher, l'affaire Lacenaire ou l'affaire Louvel autour de l'assassinat du duc de Berry illustrée remarquablement par Gilles Malandain.

L'archive n'existe que par le regard qui est posé sur elle, les questions qui lui sont soumises. Cet ouvrage est aussi étonnant au vu de la multiplicité et de l'originalité des sources et matériaux qui y sont déployés : archives, dossiers, interrogatoires, rapports médico-légaux, circulaires, presse, dossiers de procédure, lettres d'avocats, de substituts, sans oublier les écrits de juristes ou réformateurs du monde pénal et judiciaire, tels Adolphe Guillot, Adhémar Esmein, Adolphe Prins, Ernest Désiré Glasson, Gustave Le Poittevin ou Edmond Locard. Il s'agit ici d'un magnifique plaidoyer pour la richesse, la capacité d'investigation et la méthodologie du travail d'historien. Le matériau historique induit des réflexions qui peuvent être autant de nouvelles questions à poser à l'objet étudié. L'enquête judiciaire est un objet extrêmement porteur pour plusieurs champs historiographiques, mais également pluridisciplinaires (sociologie, science politique ${ }^{2}$ et anthropologie).

Cet ouvrage, hommage aux grands chantiers intellectuels collectifs, donne le sentiment d'une complétude sur le sujet, valorise la pertinence de l'historicisation sur cet objet toujours d'actualité et profondément contemporain, et donne envie que la recherche s'étende à d'autres pays et à d'autres époques. On y voit comment l'enquête judiciaire est un véritable instrument des politiques, permettant 
d'aborder la question de la circulation des savoirs, des pratiques et des modèles; une enquête judiciaire qui contribue à une analyse des mécanismes de gestion de l'ordre dans la société du $\mathrm{XIX}^{\mathrm{e}}$ siècle et à celle de ses manifestations à travers la production des normes juridiques, politiques, morales et sociales.

MARTINE KALUSZYNSKI

1 - Carlo GinZBurg, «Signes, traces, pistes. Racines d'un paradigme de l'indice », Le Débat, 6, 1980, p. 3-44.

2 - Johanna SIMEANT (sous la direction), «Le modèle de l'enquête judiciaire face aux crises extrêmes », Critique internationale, 36, 2007. 